\title{
Risk Factors of Chikungunya Outbreak in Mareka District, Southern Ethiopia, 2019: Unmatched Case Control Study
}

\author{
Tadesse Yalew Assefa ${ }^{1,2, *}$, Eyoel Berhan ${ }^{2}$, Fekadu Gemechu ${ }^{1}$, Neamin Tesfaye ${ }^{1}$ \\ ${ }^{1}$ Public Health Emergency Management Center, Ethiopian Public Health Institute, Addis Ababa, Ethiopia \\ ${ }^{2}$ Department of Public Health, Saint Paul's Hospital Millennium Medical College, Addis Ababa, Ethiopia
}

Email address:

tadesseyalew021@gmail.com (T. Y. Assefa)

${ }^{*}$ Corresponding author

\section{To cite this article:}

Tadesse Yalew Assefa, Eyoel Berhan, Fekadu Gemechu, Neamin Tesfaye. Risk Factors of Chikungunya Outbreak in Mareka District, Southern Ethiopia, 2019: Unmatched Case Control Study. International Journal of Infectious Diseases and Therapy.

Vol. 5, No. 3, 2020, pp. 64-69. doi: 10.11648/j.ijidt.20200503.15

Received: May 22, 2020; Accepted: June 4, 2020; Published: August 13, 2020

\begin{abstract}
Chikungunya is a Ribonucleic Acid (RNA) virus that belongs to the alpha virus genus of the Togaviridae family. Chikungunya is an emerging vector-borne disease of high public health significance in World Health Organization's SouthEast Asia Region. Factors associated with Chikungunya were identified. Unmatched a 1:2 case control study was conducted from 26 June-17 July, 2019. We recruited participants from health facilities and the community by structured questionnaire. Multivariable logistic regression was conducted to control confounders and to identify factors associated with Chikungunya. Entomological investigation was conducted and forty households and ten containers were surveyed to determine house and breteau indices. Fourteen serum-samples were tested by reverse transcription polymerase chain reaction (RT-PCR). A total of 94 cases were reported. Females accounted for $(59.6 \%)$ of the cases and $(52.1 \%)$ of the controls. Presence of Chikungunya case in the house hold (Adjusted odds ratio $=6.7 ; 95 \% \mathrm{CI}(3.42-13.03) ; \mathrm{P}=<0.001$ ); Water holding container ( $\mathrm{AOR}=2.85$, 95\%CI (1.37-5.91); $\mathrm{P}=0.005)$, presence of larva $(\mathrm{AOR}=2.85 ; 95 \% \mathrm{CI}(1.27-6.40) ; \mathrm{P}=0.011)$ and Short and Long sleeves $(\mathrm{AOR}=2.2 ; 95 \% \mathrm{CI}(1.144-4.267) ; \mathrm{P}=0.018)$ were the independent risk factors. House and breteau indices were $100 \%$ and $20 \%$ respectively. Eight out of fourteen samples tested were positive. The presence of Chikungunya case in the household, water holding container in or around the house, larva around the villages in favorable mosquito breeding sites and in the containers and not using long sleeve clothes were risk factors for contracting the diseases.
\end{abstract}

Keywords: Chikungunya, Mareka District, Outbreak, Risk Factors

\section{Introduction}

Chikungunya virus is an $R N A$ virus that belongs to the alpha virus genus of the Togaviridae family, the latter comprising a number of viruses that are mostly transmitted by arthropods. Infection with CHIK virus results in Chikungunya fever. The name Chikungunya derives from a root verb in the Kimakonde language meaning "that which bends up", i.e. to become contorted. The name reflects the stooped appearance of sufferers with arthralgia Chikungunya is an emerging vector-borne disease of high public health significance in WHO's South-East Asia Region [1].

In 2013, Chikungunya was found for the first time in the Americas and has spread to the Caribbean, South and Central
America, and North America [2]. For 2017, 10 countries reported 548 cases of Chikungunya, of which 461 (84.1\%) were confirmed. Of these cases, 303 cases $(55.3 \%)$ were locally acquired and $227(41.4 \%)$ were imported, while the remaining 18 cases had an unknown place of infection as in 2016 and 2015, the EU/EEA notification rate in 2017 was 0.1 cases per 100,000 population [3].

Chikungunya has a 60-70 nanometer diameter capsid and a phospholipids envelope. It is sensitive to temperatures above $58^{\circ}$ Celsius and also to desiccation. Believed to be enzootic throughout much of Africa, $C H I K$ virus probably spread to other parts of the world from this origin. African and Asian strains are reported to differ biologically with distinct lineages. Three lineages with distinct genotypic and antigenic characteristics have been identified: two phylogenetic groups, 
east-central-southern and West African groups from Africa; and the other Asian phylogroup. Isolates from the recent outbreak that started in the Indian Ocean islands belong to a distinct clade within the large east-central-southern African phylogenetic group and the isolates from the ongoing outbreaks in India are closely related to this. The different geographical genotypes exhibit differences in their transmission cycles: in Asia the virus appears to be maintained in an urban human-mosquito-human transmission cycle with Aedes aegypti and Aedes albopictus while the CHIK virus transmission in Africa involves a sylvatic cycle, primarily with Aedes furcifer, Aedes vittatus, fulgens, luteocephalus, dalzieli, etc as the vectors. A high vector density in the post-monsoon season accentuates virus transmission in Asia. The Aedes mosquitoes breed in domestic settings such as flower vases, water-storage containers, desert coolers, etc. and peri-domestic areas such as construction sites, coconut shells, discarded household junk items (vehicular tyres, plastic and metal cans, etc.). Adult mosquitoes rest in cool and shady areas in domestic and peri-domestic settings and bite humans during the daytime [1]. Human beings serve as the Chikungunya virus reservoir during epidemic periods. During inter-epidemic periods, a number of vertebrates have been implied as the reservoir. These include monkeys, rodents, birds and other vertebrates. CHIK virus causes a febrile illness in the majority of people with an incubation period of 2-4 days from the mosquito bite. Viremia persists for up to 5 days from the clinical onset. Commonest presenting features are: Fever (92\%) usually associated with Arthralgia (87\%), Backache (67\%) and, Headache (62\%) [4]. Because specific drug treatment and vaccines are not available, all control efforts should be directed against mosquitoes. It is important to maintain in-house and surrounding hygiene and sanitation in order to eliminate their breeding grounds. This is the most effective way to control the disease. However, efforts should be intensified before the transmission season, especially during and after the rainy season and at the time of an epidemic [5]. Mosquito-based surveillance is an integral component of an integrated vector management program. Mosquito based surveillance for CHIKV is very similar to that of $D E N V$; determining vector presence and estimating abundance are the primary tools for quantifying human risk. The principal functions of a mosquito-based CHIKV surveillance program are to: Determine presence or absence of Adieus. Aegypti and Ae. Albopictus in the geographic area, Identify what types of containers are producing mosquitoes and their relative importance, develop detailed maps for larval sites if Ae. Aegypti or Ae. albopictus are detected in an area, Collect mosquito population data and identify geographic areas of high abundance (high-risk), Monitor the effectiveness of vector control efforts and Collect data on mosquito infection rates during outbreaks to incriminate vectors [6]

This outbreak is detected in the routine surveillance system. The aim of this study was conducted to identify risk factors of Chikungunya and finally to recommend control and prevention measures.

\section{Method}

\subsection{Study Area and Period}

The investigation was conducted in Southern nations, nationalities and people's regional state, Mareka district, which situated 560 Kilo meter (KM) and $346 \mathrm{KM}$ from the capital city Addis Ababa and Hawasa city from the region respectively. Based on the information we get from the local authority, the district has 60,213 populations from which 9399 and 1921 are under five and under one aged population respectively. The district has a total of 3 health centers and 11 health posts which delivery health care service to the community. The district is administratively classified in to 15 Kebeles. The investigation is conducted from June 26 to July 17, 2019 and health facilities were selected based on case load.

\subsection{Study Design}

We conducted a descriptive analysis of the collected line list of cases followed by unmatched case control study with a case to control ratio of 1:2 in order to identify the possible risk factors of the outbreak. Febrile cases who fulfil the case definition of Chikungunya and epidemiologically linked cases at the health facility and controls who are residing within the same village where the cases identified during the study period were included in the study and febrile case which does not fulfill the cases definitions or not linked epidemiologically and controls not from the same village or area were excluded from the study during the study period.

\subsection{Sample Size}

We included all 94 cases reported during the study period and using a 1:2 case to control ratio we selected 188 controls randomly from the same village where cases are identified.

Selection of cases and controls: We recruited all Chikungunya cases at health facility as well as those identified from the community by active searching during the study period. And cases treated before the study period were excluded due to information bias during data collection and easily inaccessibility of those cases and controls were taken randomly from the same area where cases are identified.

\subsection{Data Collection Tools and Procedures}

Document review: Outpatient medical logbooks and medical record of cases at the health facility were reviewed. The laboratory findings of the first 14 cases at the national reference laboratory at EPHI were also reviewed.

Interviewing cases and controls: Using a structured questionnaire (annexed), cases and controls were interviewed by four data collectors (including the principal investigator).

\subsection{Entomological Investigation}

We had also conducted entomological investigation in one of the selected kebeles specifically in Gendo bacho kebele where most of the cases were treated and reported and 40 selected households were addressed in Gendo bacho kebele 
centering the cases reported for the patients admitted and both the natural and artificial breeding containers were targeted during the investigation. Larvae and pupae were sampled from the domestic and peri-domestic environments in order to estimate risk indices. Adult mosquitoes were collected by human landing collection using standard aspirator from the volunteer residents of household during day times.

Different artificial water holding containers have been identified like plant leaf axils, tree holes, discarded appliances, used tyres, animal water containers, potted plants and broke house equipment, flower pots, jars, open Highland water containers, pots, and bigger water holding rottos (water storage tanks) were observed to be among the commonly found appliances in the area. House Index (HI) and Breteau Index (BI) was computed by the WHO Index formulas [7].

Breteau index $=($ Number of positive containers $/$ Number of houses inspected) X 100

House index $=($ Number of infested houses $/$ Number of houses inspected) X 100

Container inde $x=($ Number of positive containers $/$ Number of containers inspected) X 100

\subsection{Data Analysis and Data Quality Assurance}

Before the start of data collection, a half-day orientation was given for data collectors. Each completed questionnaire was daily reviewed by the principal investigator to monitor the data quality. Before analysis, data was also cleaned for any missing and logically inconsistent values. All collected data are entered and analyzed using statistical software's SPSS, bivariate and multivariate (using logistic regression model) analysis are conducted using SPSS and MS excel 2013. Results were displayed using texts, tables and graphs and statistical significance was interpreted using Odd ratio with $95 \%$ confidence interval and $\mathrm{P}$ value $<0.05$.

\subsection{Laboratory Methods}

Three main laboratory tests are used for diagnosing
Chikungunya fevers: virus isolation, serological tests and molecular technique of polymerase chain reaction (PCR). Specimen is usually blood or serum but in neurological cases with meningo-encephalitic feature CSF may also be sent as specimen [1]. Samples were collected to identify the cause of the outbreak. The outbreak was tested first by rapid diagnostic test and then by RT-PCR was performed for confirmation.

\subsection{Ethical Clearance}

Ethical clearance was obtained from Ethiopian Public Health Institute (EPHI). A letter was written for District health offices in order to obtain approval on data collection and approval of the health facilities administration was obtained before approaching the patients. Informed verbal consent was obtained from all study participants before conducting interview by explaining the purpose of the study. Privacy and confidentiality was ensured. The name of respondents was not written on the questionnaire, therefore, the information study participants provide was not known to others. The participation of individuals in this study was purely voluntary.

\section{Result}

\section{Socio Demographic Characteristics}

A total of 94 case patients and 188 controls were included in the study. From the total study participants, 128 (45\%) were males and $154(55 \%)$ were females. Out of the 94 cases, $38(40.4 \%)$ were males. The mean age for cases and controls were 29 and 30 respectively. From the total cases, 23 (24.5\%) were singles and $60(63.8 \%$ were married. From the total cases, $37(39.4 \%)$ were farmers and from the controls 53 $(28.2 \%)$ were farmers and $37(19.7 \%)$ were house wife's. From the cases, $30(31.9 \%)$ were illiterate and more than half the cases $49(52.1 \%)$ had finished primary school. Whereas for the controls 105 (55.9\%) had finished primary school and $8(4.3 \%)$ were reached college and university (Table 1$)$.

Table 1. Demographic characteristics on Chikungunya virus cases and controls, Mareka District, 2019.

\begin{tabular}{|c|c|c|c|c|c|c|}
\hline \multirow{2}{*}{\multicolumn{2}{|c|}{ Descriptive Variables }} & \multirow{2}{*}{$\begin{array}{l}\text { Cases } \\
\text { Number }\end{array}$} & \multirow{3}{*}{$\frac{\%}{40.4}$} & \multicolumn{2}{|l|}{ Controls } & \multirow{2}{*}{ Total $n=282$} \\
\hline & & & & Number & $\%$ & \\
\hline \multirow{2}{*}{ Sex } & Male & 38 & & 90 & 47.9 & $128(45 \%)$ \\
\hline & Female & 56 & 59.6 & 98 & 52.1 & $154(55 \%)$ \\
\hline \multirow{4}{*}{ Age } & $0 \_4$ & 2 & 2.1 & 0 & 0 & $2(0.7 \%)$ \\
\hline & $5 \_14$ & 17 & 18.1 & 13 & 6.9 & $30(10.6 \%)$ \\
\hline & $15 \_44$ & 67 & 71.3 & 155 & 82.4 & $222(78.7 \%)$ \\
\hline & $45+$ & 8 & 8.5 & 20 & 10.6 & $28(9.9 \%)$ \\
\hline \multirow{4}{*}{ Marital status } & Single & 23 & 24.5 & 43 & 22.9 & $66(23.4 \%)$ \\
\hline & Married & 60 & 63.8 & 129 & 68.6 & $189(67.0 \%)$ \\
\hline & Divorced & 0 & 0 & 4 & 2.1 & $4(1.4 \%)$ \\
\hline & NA & 11 & 11.7 & 12 & 6.4 & $23(8.2 \%)$ \\
\hline \multirow{4}{*}{ Educational status } & Illiterate & 30 & 31.9 & 49 & 26.1 & $79(28.0 \%)$ \\
\hline & Primary school & 49 & 52.1 & 105 & 55.9 & $154(54.6 \%)$ \\
\hline & Secondary school & 13 & 13.8 & 26 & 13.8 & $39(13.8 \%)$ \\
\hline & College and university & 2 & 2.1 & 8 & 4.3 & $10(3.5 \%)$ \\
\hline
\end{tabular}




\begin{tabular}{lllllll}
\hline \multirow{2}{*}{ Descriptive Variables } & & Cases & & Controls & \\
\cline { 3 - 6 } & & Number & $\mathbf{\%}$ & Number & Total n=282 \\
\hline & Student & 19 & 20.2 & 65 & 34.6 & $84(29.8 \%)$ \\
& Daily laborer & 6 & 6.4 & 11 & 5.9 & $17(6.0 \%)$ \\
Occupation & Merchant & 3 & 3.2 & 15 & 8 & $18(6.4 \%)$ \\
& Farmer & 37 & 39.4 & 53 & 28.2 & $90(31.9 \%)$ \\
& House wife & 27 & 28.7 & 37 & 19.7 & $64(22.7 \%)$ \\
Mean age & Government & 2 & 2.1 & 7 & 3.7 & $9(3.2 \%)$ \\
\end{tabular}

\section{Laboratory Result}

A total of fourteen samples were collected and sent to EPHI national reference laboratory from Gendo kebele of Mareka district for confirmation of the outbreak. From the total samples eight were tested positive for Chikungunya virus by molecular techniques of PCR with a positivity rate of $(57.1 \%(8 / 14))$ and the rest were tested negative for the virus.

\section{Entomological investigation}

Enset leaf axil holds the lion's share in the breeding of Aedes mosquitoes as all the assessed Enset surrounded HHs have seen to be larvae and pupa positive.

Eight types of positive containers have been identified positive out of ten types of containers assessed from 40 households. House, Breteau and container were 100\%, 20\% and $80 \%$ respectively (Table 2 ).

Table 2. Entomological larval and pupae surveyed in selected households of Gendo bacho kebele, 2019.

\begin{tabular}{lllllll}
\hline \multicolumn{2}{l}{ Place investigated } & Type of vector & Vector indices & & \\
\cline { 4 - 6 } Woreda & Kebele & collected & Breteau Index & House index & Container index \\
\hline Mareka & Gendo bacho & Aedes species & $20 \%$ & $100 \%$ & $80 \%$ & Yes/No \\
\hline
\end{tabular}

\section{Knowledge towards CHIKV}

The participants were also asked knowledge questions regarding CHIKV and out of the total case respondents, 26 (27.7\%) of them had heard about CHIKV and $56(29.9 \%)$ of controls had incite about the diseases. Only $10(10.6 \%)$ and $17(9 \%)$ cases and controls respectively state that the causative agent for Chikungunya is a virus. Among the cases and controls, $19(20.2 \%)$ and $32(17 \%)$ of the case and controls respectively state mosquito as a means of transmission. On the other hand $59(62.8 \%)$ of the cases and 143 (76.15) of the controls had no idea about the time mosquito bites. Of the cases $19(20.2 \%)$ and from the controls $47(25 \%)$ know about the symptoms, even if more than half of the cases and controls did not heard about the disease (Table 3).

Table 3. Knowledge on Chikungunya cases and controls, Mareka District, 2019.

\begin{tabular}{|c|c|c|c|}
\hline Characteristics & Cases & Controls & Total $n=282$ \\
\hline \multicolumn{4}{|c|}{ Heard About Chikungunya Virus } \\
\hline Yes & $26(27.7 \%)$ & $56(29.8 \%)$ & $82(29.1 \%)$ \\
\hline No & $68(72.3 \%)$ & $132(70.2 \%)$ & $200(70.9 \%)$ \\
\hline \multicolumn{4}{|c|}{ Cause Of Chikungunya } \\
\hline Virus & $10(10.6 \%)$ & $17(9 \%)$ & $27(9.6 \%)$ \\
\hline Don't Know & $84(89.4 \%)$ & $171(91 \%)$ & $255(90.4 \%)$ \\
\hline By Mosquito & $19(20.2 \%)$ & $32(17 \%)$ & $51(18.1 \%)$ \\
\hline Air Droplets & $4(4.3 \%)$ & $17(9 \%)$ & $21(7.4 \%)$ \\
\hline Don't Know & $71(75.5 \%)$ & $139(73.9 \%)$ & $210(74.5 \%)$ \\
\hline \multicolumn{4}{|c|}{ Time Mosquito Bites } \\
\hline Night & $10(10.6 \%)$ & $19(10.1 \%)$ & $29(10.3 \%)$ \\
\hline Day & $25(26.6 \%)$ & $26(13.8 \%)$ & $51(18.1 \%)$ \\
\hline Don't Know & $59(62.8 \%)$ & $143(76.1 \%)$ & $202(71.6 \%)$ \\
\hline Yes & $17(18.1 \%)$ & $43(22.9 \%)$ & $60(21.3 \%)$ \\
\hline No & $18(19.1 \%)$ & $32(17 \%)$ & $50(17.7 \%)$ \\
\hline Do Not Know & $59(62.8 \%)$ & $113(60.1 \%)$ & $172(61 \%)$ \\
\hline \multicolumn{4}{|c|}{ Know Symptoms Of Chikungunya } \\
\hline Yes & $19(20.2 \%)$ & $47(25 \%)$ & $66(23.4 \%)$ \\
\hline No & $75(79.8 \%)$ & $141(75 \%)$ & $216(76.6 \%)$ \\
\hline
\end{tabular}

Sign and Symptoms towards CHIKV

The cases were also asked for sign and symptoms and fever $(100 \%)$, headache $(100 \%)$ and muscle and joint pain (94\%) were the top identified symptoms (Table 4). 
Table 4. Distribution of sign and symptoms of Chikungunya cases, Mareka, District, 2019.

\begin{tabular}{lll}
\hline Symptoms & $\begin{array}{l}\text { Number } \\
\text { of cases }\end{array}$ & $\begin{array}{l}\text { Proportion of all } \\
\text { cases (\%) }\end{array}$ \\
\hline Fever & 94 & 100.0 \\
Headache & 94 & 100.0 \\
Restlessness or lethargy & 7 & 7.4 \\
Abdominal pain, nausea or vomiting & 13 & 13.8 \\
Chills & 84 & 89.4 \\
Muscle and joint pain & 88 & 94.0 \\
\hline
\end{tabular}

\section{Risk factors of CHIKV}

On bivariate analysis, using LLINs while sleeping $(\mathrm{P}=0.023 ; \mathrm{OR}=0.28 ; 95 \% \mathrm{CI} \quad(0.096-0.838))$, presence of water holding container in/around the house $(\mathrm{P}=0.041 ; \mathrm{OR}=2$;
95\%CI (1.03-3.76)), presence of larva $(\mathrm{P}=0.044 ; \mathrm{OR}=2.1$; $95 \% \mathrm{CI}(3.68-12.86))$, travel history within the last two weeks $(\mathrm{P}=0.006 ; \mathrm{OR}=2.17 ; 95 \% \mathrm{CI}(1.25-3.748))$, type of clothe $(\mathrm{P}=0.023 ; \mathrm{OR}=0.28 ; 95 \% \mathrm{CI} \quad(1.84-6.233))$ and presence of diseased person $(\mathrm{P}=<0.001 ; \mathrm{OR}=6.88 ; 95 \% \mathrm{CI}(3.68-12.86))$ were important risk factors identified (Table 4).

On multivariable analysis, the factor remained statically significantly associated with illness were; presence of Chikungunya case in the house hold (Adjusted $\mathrm{OR}=6.7$; 95\%CI (3.42-13.03); $\mathrm{P}=<0.001$ ); Water holding container in/around the house (Adjusted $\mathrm{OR}=2.85,95 \% \mathrm{CI}(1.37-5.91)$; $\mathrm{P}=0.005$ ), presence of larva (adjusted $\mathrm{OR}=2.85 ; 95 \% \mathrm{CI}$ $(1.27-6.40) ; \mathrm{P}=0.011)$ and type of cloth (Adjusted $\mathrm{OR}=2.2$; 95\%CI (1.144-4.267); $\mathrm{P}=0.018$ ) (Table 5).

Table 5. Bivariate analysis for risk factors for Chikungunya virus, Mareka District, 2019.

\begin{tabular}{|c|c|c|c|c|c|}
\hline Variables & Category & Cases & Controls & COR $(95 \% \mathrm{CI})$ & P-value \\
\hline \multirow{2}{*}{ Sex } & Male & $38(40.4 \%)$ & $90(47.9 \%)$ & $1.35(0.82-2.24)$ & 0.237 \\
\hline & Female & $56(59.6 \%$ & $98(52.1 \%)$ & & \\
\hline \multirow{2}{*}{ Presence of diseased person } & Yes & $41(43.6 \%)$ & $19(10.1 \%)$ & $6.88(3.68-12.86)$ & $<0.001$ \\
\hline & No & $53(56.4 \%)$ & $169(89.9 \%$ & $* 1 *$ & \\
\hline \multirow{2}{*}{$\begin{array}{l}\text { Presence of water holding container } \\
\text { in/around the house }\end{array}$} & Yes & $73(77.7 \%)$ & $164(87.2 \%)$ & $2(1.03-3.76)$ & 0.041 \\
\hline & No & $21(22.3 \%)$ & $24(12.8 \%)$ & $* 1 *$ & \\
\hline \multirow{2}{*}{ Presence of larva in the container } & Yes & $17(18.1 \%)$ & $18(9.6 \%)$ & $2.1(1.02-4.26)$ & 0.044 \\
\hline & No & $77(81.9 \%)$ & $170(90.4 \%)$ & $* 1 *$ & \\
\hline \multirow{2}{*}{ Having LLINs } & Yes & $20(21.3 \%)$ & $46(24.5 \%)$ & $0.83(0.46-1.513)$ & 0.551 \\
\hline & No & $74(78.7 \%)$ & $142(75.5 \%)$ & $* 1 *$ & \\
\hline \multirow{2}{*}{ Using LLINs while sleeping in daytime } & Yes & $7(33.3 \%)$ & $30(63.8 \%)$ & $0.28(0.096-0.838)$ & 0.023 \\
\hline & No & $14(66.7 \%)$ & $17(36.2 \%)$ & $* 1 *$ & \\
\hline \multirow{2}{*}{ Stagnant water in the village } & Yes & $7(7.4 \%)$ & $5(2.7 \%)$ & $* 1 *$ & \\
\hline & No & $87(92.6 \%)$ & $183(97.3 \%)$ & $0.34(0.105-1.1)$ & 0.072 \\
\hline \multirow{2}{*}{$\begin{array}{l}\text { Close contact with person with same } \\
\text { complaint within the last one to two weeks }\end{array}$} & Yes & $30(31.9 \%)$ & $40(21.3 \%)$ & $1.73(0.994-3.03)$ & 0.053 \\
\hline & No & 64 (68.1\%) & $148(78.7 \%)$ & $* 1 *$ & \\
\hline \multirow{2}{*}{ Travel history within the last two weeks } & Yes & $34(36.2 \%)$ & $39(20.7 \%)$ & $2.17(1.25-3.748)$ & 0.006 \\
\hline & No & $60(63.8 \%)$ & $149(79.3 \%)$ & $* 1 *$ & \\
\hline \multirow{2}{*}{ Type of clothes (Short and Long sleeves) } & Short sleeves & $78(83 \%)$ & $111(59 \%)$ & $3.38(1.84-6.233)$ & $<0.001$ \\
\hline & Long sleeves & $16(17 \%)$ & $77(41 \%)$ & $* 1 *$ & \\
\hline
\end{tabular}

Table 6. Multivariable analysis for different variables. Mareka, District, 2019.

\begin{tabular}{lllll}
\hline Exposure & AOR & 95\%Confidence interval & P-value & S. E \\
\hline Presence of Chikungunya case in the house hold & 6.7 & $(3.42-13.03)$ & $<0.001$ & 0.34 \\
Water holding container in/around the house & 2.85 & $(1.37-5.91)$ & 0.005 & 0.37 \\
Presence of larva & 2.85 & $(1.27-6.40)$ & 0.011 & 0.41 \\
Type of clothes (Short sleeve and Long sleeve) & 2.2 & $(1.144-4.267)$ & 0.018 & 0.34 \\
\hline
\end{tabular}

\section{Discussion}

This investigation revealed that the Chikungunya virus outbreak was occurred for the first time in Mareka district of southern nations, nationalities and peoples region. Environmental factors and community behaviors play a significant role in Chikungunya outbreak and spread. Chikungunya is not a notifiable disease. However, depending upon the spread, countries in the Region may make it mandatory for the primary health centers, other clinics and hospitals, both in the public and private sectors to notify suspected cases to authorities. Occurrence of clustering of cases compatible with the definition of a suspected case should indicate a possible outbreak and this should immediately be reported to the nearest health authority [1]. In this outbreak, most of the cases had symptoms of fever, headache, joint pains and abdominal pain, nausea and vomiting in few patient (13.8\%).

The outbreak finding revealed that Chikungunya infection occurred equally between the genders and age groups. This study is consistent with a study conducted in Kedougou, Southeastern Senegal [8]. All cases of Chikungunya infection had fever, headache and joint pain (100\%). A prospective study in a tertiary center conducted in north India had a similar findings [9]. This study shows that, males were more affected than females. This may be the reason due to that males spent most of their time outside of the house working in farming area which may be consistent to the nature of the adult female mosquito that bites during day time [1].

The study revealed that; Presence of Chikungunya case in the house hold, availability of stagnant water in or around the 
house, presence of larva around the villages in favorable mosquito breeding sites (in the containers), using LLINS while sleeping during day time as protective and not using long sleeve clothes were the risk factors contracting the Chikungunya virus infection. Those who had water holding containers in or around their houses were 2.9 times more at risk to have the diseases. In consistent to this; results of a case control study carried out in South India identified that those having water containers like storing of water for drinking and washing/bathing, use of plastic buckets and cement barrels for storing water, not covering the water storage container, tyres, broken plastic vessels or plates in the backyard of the houses were identified as a risk of acquiring the CHIKV infection [10]. House, Breteau and container were $100 \%, 20 \%$ and $80 \%$ respectively, and all were above the national standards of $>=5 \%$ and $>=20 \%$ respectively and these high indices indicates the abundance of breeding habitats for Aedes mosquitoes and also signifies that the place is sensitive and vulnerable to disease transmission [6]. The study also showed a significant association between CHIKV infection and not using clothes that fully cover. Not using clothes that fully cover is 2.2 times to acquire the disease than those using full body cover which is consistent with a case control study carried out in South India [10].

The current study also revealed that a significant association between CHIKV infection and presence of diseased person with in the family. This study showed that; there is a significant association between CHIKV infection and a Chikungunya case (presence of diseased person) in the family or house hold. Having a Chikungunya case in the house hold was 6.7 times risk for acquiring the disease. This study has similar findings with a case control study conducted in Laguna, Philippines, 2012 [11].

The investigation had some limitations. Even though there was full response rate in this study, we interviewed only patients that appeared to health facilities and so may have missed factors related to cases that did not seek medical care.

\section{Conclusion}

This is the first outbreak of Chikungunya in the district. All cases were recovered from the illness and there was no death occurred. The presence of Chikungunya case in the household, presence of water holding container in or around the house, presence of larva around the villages in favorable mosquito breeding sites or in the containers and not using long sleeve clothes were independent risk factors for disease contraction. Vector control and community mobilization and awareness creation plays a significant role

What is already known on this topic?

1) Chikungunya fever is highly contagious

2) Chikungunya virus is a vector borne diseases

3) Aedes Mosquitoes are responsible for transmission of

\section{Chikungunya Virus}

What this study adds?

Risk factors for Chikungunya Virus infection

Conflict of interest

The authors declare that they have no competing interests. Author's contribution

TY design the study, participate in field investigation, conduct analysis and write the manuscript. EB participate in designing the study and manuscript writing. FG involved in entomological and field investigation. NT participate in data analysis. All authors review the manuscript and approve the submission.

\section{Acknowledgements}

Our sincere thanks go to sincerely appreciate the contributions made during the outbreak investigation, by Regional Health bureau, Dawro Zonal health department, Mareka District health office and the community members. No fund was provided for this work.

\section{References}

[1] Guidelines for Prevention \& Control of Chikungunya Fever.

[2] Asia S, America C. Chikungunya Virus : What you need to know. 2015.

[3] Report S. Chikungunya virus disease Annual Epidemiological Report for 2017 Key facts. 2019;(April).

[4] Guidelines on Clinical Management of Chikungunya Fever.

[5] Chikungunya fever.

[6] For C, Control D. Chikungunya: Vector Surveillance and Control in the United States.

[7] Mnzava A, Pinzon MQ. Entomological surveillance for Aedes spp. in the context of Zika virus Interim guidance for entomologists.

[8] Sow A, Faye O, Diallo M, Diallo D, Chen R, Faye O, et al. Chikungunya Outbreak in Kedougou, Southeastern Senegal in 2009 - 2010. 2010.

[9] Dinkar A, Singh J, Prakash P, Das A, Nath G. Journal of Infection and Public Health Hidden burden of chikungunya in North India; A prospective study in a tertiary care centre. J Infect Public Health [Internet]. 2018; 11 (4): 586-91. Available from: http://dx.doi.org/10.1016/j.jiph.2017.09.008.

[10] Ramachandran V, Manickam P, Kaur P, Murhekar M V, Kanagasabai K, Jeyakumar A, et al. iMedPub Journals Behavioural Determinants Associated with CHIKV Outbreak in Gouriepet, Avadi, Chennai, South India Abstract Study setting. 2015; 1-6.

[11] Report OI. Investigation of chikungunya fever outbreak in Laguna, Philippines, 2012. 2015; 6 (3): 8-11. 